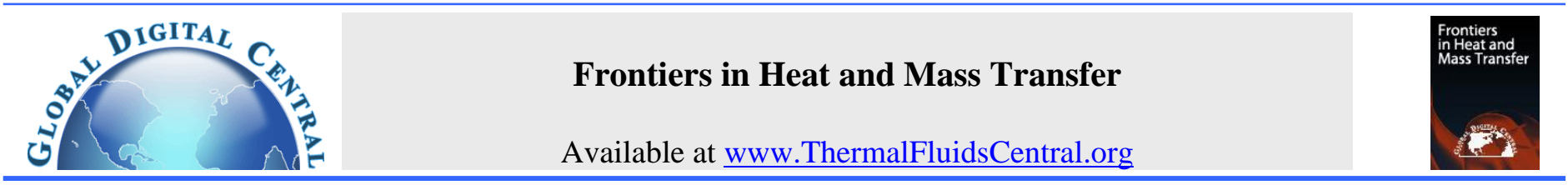

\title{
SCALING GROUP TRANSFORMATION FOR MIXED CONVECTION IN A POWER-LAW FLUID SATURATED POROUS MEDIUM WITH EFFECTS OF SORET, RADIATION AND VARIABLE PROPERTIES
}

\author{
J. Pranitha ${ }^{\mathrm{a}, *}$, G. Venkata Suman ${ }^{\mathrm{a}}$, D. Srinivasacharya ${ }^{\mathrm{a}}$ \\ ${ }^{a}$ Department of Mathematics, National Institute of Technology Warangal-506004, Telangana, India
}

\begin{abstract}
An analysis is performed to investigate the influence of radiation, thermal-diffusion and variable properties on mixed convection flow, heat and mass transfer from a vertical plate in a porous medium saturated with a power-law fluid. The non-linear partial differential equations are reduced to ordinary differential equations by implementing Lie scaling group transformations. These ordinary differential equations are solved numerically by implementing a shooting technique. The numerical results for dimensionless velocity, temperature and concentration profiles for pseudo-plastic, Newtonian and dilatant fluids are presented graphically for different values of variable viscosity, variable thermal conductivity, Soret and radiation parameters. Heat and mass transfer results are presented in tabular form. The present numerical results are compared with previously published work and the results are found to be in excellent agreement.

Keywords: Mixed convection, Variable viscosity, Power-law fluid, Thermal Radiation, Thermal conductivity, Soret, Lie Scaling Group Transformations.
\end{abstract}

\section{INTRODUCTION}

Research on convective heat and mass transfer of power-law fluids has received much attention for several years due to its theoretical and practical importance in industrial processing equipment, dealing with molten plastics, paints, polymers, pharmaceutical formulations, cosmetics and toiletries, ground water flow, etc. Several researchers have presented their work in the literature focusing on the problem of convection heat and mass transfer in power-law fluids. Details of review of the literature on power-law fluids and their applications may be found in the recent books by Pop and Ingham (2001), Nield and Bejan (2006) and Vafai (2015). On the other hand, Power-law fluid saturated porous media are a branch of research undergoing rapid growth in fluid mechanics and heat transfer characteristic of a wide range of engineering applications in several problems areas such as nuclear reactors, foodstuffs, thermal insulations, polymeric liquids and geothermal systems etc. Analytical and numerical investigation of horizontal line heating sources in a power-law fluid saturated porous medium has been discussed by Nakayama (1993). Kairi et al. (2009) carried out interesting applications of non-Darcy natural convective heat and mass transfer in a power-law fluid under the influence of thermal and solutal dispersions. Soret and Dufour effects on natural convection of a power-law fluid over a vertical plate embedded in a porous medium were reported by Srinivasacharya et al. (2012).

All the above authors assumed that the thermophysical properties of the fluid are constant. However, it is well known that these variable properties may change with temperature, especially for fluid viscosity and thermal conductivity. These variable properties are very important in several engineering and industrial applications such as volumetric solar receivers, foam insulations, boilers, heat transfer in furnaces, fibrous and porous burners etc. Hassanien et al. (2003) expressed mixed convection along a wedge embedded in a fluid saturated porous medium for the cases of uniform heat flux and uniform mass flux by using the effect of variable viscosity and thermal conductivity. The influence of radiation and variable properties of heat transfer from a moving surface in a micropolar fluid through a porous medium was discussed Elsayed et al. (2004). Seddeek and Salama (2007) examined unsteady hydromagnetic heat transfer past a semi-infinite vertical porous moving plate with variable suction taking variable viscosity and thermal conductivity effects into account. Jayanthi and Kumari (2007) investigated numerically both free and mixed convection flow on a vertical surface in a non-Newtonian fluid saturated porous medium in the presence of temperature-dependent viscosity. Mahanti and Gaur (2009) obtained similarity solutions for natural convection flow of an incompressible viscous fluid with influence of a heat sink along an isothermal vertical surface using variable properties. Srinivasacharya et al. (2015) obtained a similarity solution to explore the effects of Soret and Dufour on mixed convective flow along a vertical wavy surface in a porous medium with variable properties. Rao et al. (2017) investigated numerically the influence of thermal radiation on MHD flow over a linearly stretching sheet in a porous medium with

${ }^{\dagger}$ Corresponding author.Email: pranithaj@gmail.com,gvsuman1@gmail.com 


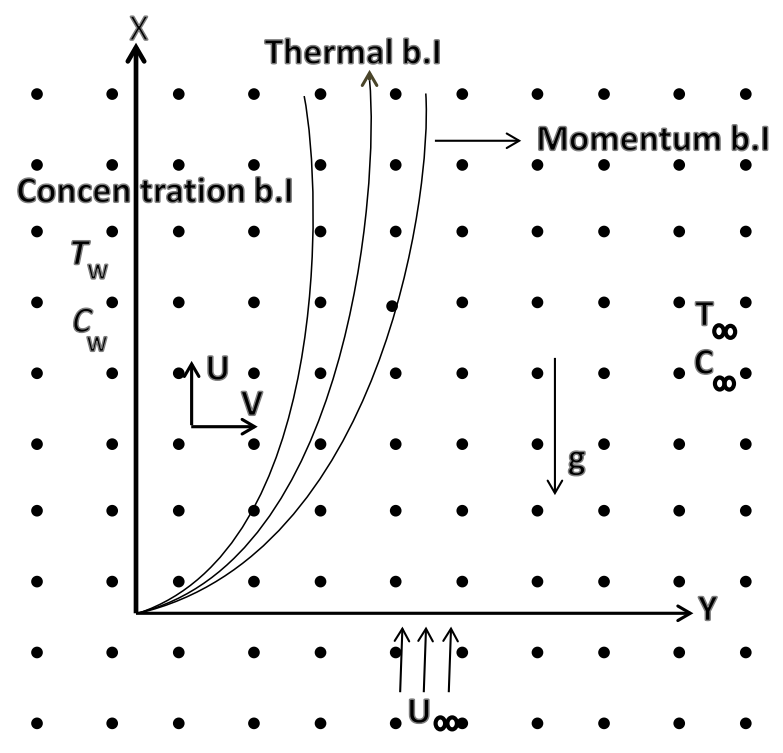

Fig. 1 Physical model

variable viscosity and thermal conductivity.

Thermal radiation on mixed convective boundary layer flow has received great attention because of very important application such as solar power technology, space technology, astrophysical flows and electrical power generation, etc. Grosan and Pop (2006) discussed numerically the influence of radiation free convective flow over a vertical surface in a power-law fluid. Hayat et al. (2011) focused on two dimensional mixed convection stagnation-point flow of power-law fluids towards a stretching sheet with the effect of MHD and radiation. Gbadeyan et al. (2011) considered the problem of magnetic field, Soret and Dufour effects on mixed convection flow over a stretching vertical surface in a viscoelastic fluid saturated porous medium. Mahmoud and Megahed (2013) expressed the influence of radiation, Dufour and Soret on mixed convective flow in a study related to laminar flow along a vertical surface. Srinivasacharya et al. (2014) reported mixed convection flow in a doubly stratified fluid saturated porous medium in the presence of Dufour and Soret effects.

In this paper, we introduce the Lie group analysis, namely scaling group transformations for mixed convection boundary layer problems. The main advantages of these Lie group transformations is to obtain similarity transformations for the system of nonlinear partial differential equations. Moreover, the Lie Group technique has been applied by many researchers (see Oberlack (1999), (Kandasamy et al., 2010), (Afify et al., 2014), (Ferdows et al., 2013) and (Uddin et al., 2015)). Recently, ElAziz and Afify (2016) reported Lie group analysis for a power-law fluid over a stretching sheet with effect of magnetohydrodynamic and variable properties. To the best of the authors' knowledge, the study related to Lie group analysis for a power-law fluid with variable properties has not been explained in the literature. The main aim of this work is to express the influence of Soret, variable properties and radiation on mixed convection flow over a vertical surface embedded in a porous medium saturated with a power-law fluid by using Lie scaling group transformations.

\section{MATHEMATICAL FORMULATION}

Consider steady, incompressible, laminar, mixed convection heat and mass transfer boundary layer flow over a vertical surface embedded in a powerlaw fluid saturated porous medium. Choose the two dimensional coordinate system such that the $\bar{X}$-axis is along the vertical plate and $\bar{Y}$-axis normal to the plate. The physical model and coordinate system are shown in Fig (1). The plate is maintained at a constant temperature $\bar{T}_{w}$ and concentration $\bar{C}_{w}$. The ambient temperature is $\bar{T}_{\infty}$ and the concentration is $\bar{C}_{\infty}$. Applying the boundary layer assumption and Boussinesq approximations, the governing equations are the mass, momentum, energy and concentration equations which may be written as:

$$
\begin{gathered}
\frac{\partial \bar{U}}{\partial \bar{X}}+\frac{\partial \bar{V}}{\partial \bar{Y}}=0 \\
n \bar{U}^{n-1} \frac{\partial \bar{U}}{\partial \bar{Y}}=\frac{\partial}{\partial \bar{Y}}\left[\frac{K g \rho_{\infty}}{\mu}\left(\beta_{T}^{*}\left[\bar{T}-\bar{T}{ }_{\infty}\right]+\beta_{C}^{*}\left[\bar{C}-\bar{C}_{\infty}\right]\right)\right] \\
\bar{U} \frac{\partial \bar{T}}{\partial \bar{X}}+\bar{V} \frac{\partial \bar{T}}{\partial \bar{Y}}=\frac{\partial}{\partial \bar{Y}}\left[\alpha \frac{\partial \bar{T}}{\partial \bar{Y}}-\frac{1}{\rho C_{p}} q_{r}\right] \\
\bar{U} \frac{\partial \bar{C}}{\partial \bar{X}}+\bar{V} \frac{\partial \bar{C}}{\partial \bar{Y}}=D_{m} \frac{\partial^{2} \bar{C}}{\partial \bar{Y}^{2}}+\frac{D_{m} K_{T}}{T_{m}} \frac{\partial^{2} \bar{T}}{\partial \bar{y}^{2}}
\end{gathered}
$$

where $\bar{X}$ and $\bar{Y}$ are the Cartesian coordinates, $\bar{U}$ and $\bar{V}$ are the velocity components in the stream wise $\bar{X}$ and cross-stream $\bar{Y}$ directions, respectively, $\beta_{T}^{*}$ represents the thermal expansion coefficient and $\beta_{C}^{*}$ represents the concentration coefficient, $\bar{T}$ indicates the temperature, $\bar{C}$ indicates the concentration, $K$ represents the permeability, $K_{T}$ indicates the thermal diffusion ratio, $\alpha$ represents the thermal conductivity, $D_{m}$ is the mass diffusivity of the saturated porous medium, $T_{m}$ is the mean fluid temperature, $C p$ represents the specific heat capacity, $q_{r}$ is the component of radiative heat flux, $n$ is the index in the power-law variation of viscosity. $n<1$ for a pseudo-plastic fluid, $n>1$ for a dilatant fluid and $n=1$ for a Newtonian fluid.

The boundary conditions are

$$
\begin{aligned}
& \bar{V}=0, \quad \bar{T}=\bar{T}_{w}, \quad \bar{C}=\bar{C}_{w} \quad \text { at } \quad \bar{Y}=0 \\
& \bar{U}=U_{\infty}, \quad \bar{T}=\bar{T}_{\infty}, \quad \bar{C}=\bar{C}_{\infty} \quad \text { as } \quad \bar{Y} \rightarrow \infty
\end{aligned}
$$

The viscosity $\mu$ of the fluid is considered to be an inverse function of temperature and it can be expressed as ( Lai and Kulacki (1990))

$$
\frac{1}{\mu}=\frac{1}{\mu_{\infty}}\left[1+\gamma^{*}\left(\bar{T}-\bar{T}_{\infty}\right)\right] \quad \text { i.e } \quad \frac{1}{\mu}=b\left(\bar{T}-\bar{T}_{e}\right)
$$

where $b=\frac{\gamma^{*}}{\mu_{\infty}}, \bar{T}_{e}-\bar{T}_{\infty}=-\frac{1}{\delta}, \mu_{\infty}$ is the coefficient of viscosity and both $b, \bar{T}_{e}$ are constants and their values depend on the reference state and the small parameter $\gamma^{*}$, reflecting a thermal property of the fluid.

Variable thermal conductivity $\alpha$ which appears in Eq.(3) in the nondimensional form Slattery (1972) can be written as

$$
\alpha=\alpha_{0}(1+\bar{T} \beta) .
$$

where $\beta$ is the thermal conductivity.

The quantity $q_{r}$ is the radiative heat flux which is simplified by using the Rosseland diffusion approximation for an optically thick fluid. Thus

$$
q_{r}=\frac{-4 \sigma^{*}}{3 k^{*}} \frac{\partial \bar{T}^{4}}{\partial \bar{y}},
$$

where $\sigma^{*}$ is the Stefan-Boltzmann constant and $k^{*}$ is the Rosseland mean absorption coefficient.

Now, we introduce the following dimensionless variables:

$$
\left.\begin{array}{l}
X=\frac{\bar{X}}{L}, \quad Y=\frac{\bar{Y}}{L} P e^{\frac{1}{2}}, \quad U=\frac{\bar{U} L}{\alpha_{0} P e}, \quad V=\frac{\bar{V} L}{\alpha_{0} P e^{\frac{1}{2}}} \\
T(\eta)=\frac{\bar{T}-\bar{T}_{\infty}}{\bar{T}_{w}-\bar{T}_{\infty}}, \quad C(\eta)=\frac{\bar{C}-\bar{C}_{\infty}}{\bar{C}_{w}-\bar{C}_{\infty}}
\end{array}\right\}
$$


In the view of the continuity Eq. (1) we introduce a stream function $\psi(X, Y)$ as

$$
U=\frac{\partial \psi}{\partial Y}, \quad V=-\frac{\partial \psi}{\partial X}
$$

Substituting Eqs. (6) - (10) into Eqs. (2) - (4), leads to the following momentum, energy and concentration equations

$$
\begin{array}{r}
\Delta_{1}=n\left(\frac{\partial \psi}{\partial Y}\right)^{n-1} \frac{\partial^{2} \psi}{\partial Y^{2}}-\lambda^{n} \\
\left(\frac{\partial T}{\partial Y}+B \frac{\partial C}{\partial Y}\right)\left(1-\frac{T}{\theta_{e}}\right) \\
+\frac{\lambda^{n}}{\theta_{e}}(T+B C)\left(\frac{\partial T}{\partial Y}\right)=0,
\end{array}
$$

$\Delta_{2}=\frac{\partial \psi}{\partial Y} \frac{\partial T}{\partial X}-\frac{\partial \psi}{\partial X} \frac{\partial T}{\partial Y}-\beta\left(\frac{\partial T}{\partial Y}\right)^{2}-\left(1+T \beta+\frac{4 R}{3}\right) \frac{\partial^{2} T}{\partial Y^{2}}=0$

$$
\Delta_{3}=\frac{\partial \psi}{\partial Y} \frac{\partial C}{\partial X}-\frac{\partial \psi}{\partial X} \frac{\partial C}{\partial Y}-\frac{1}{L e} \frac{\partial^{2} C}{\partial Y^{2}}-S r \frac{\partial^{2} C}{\partial Y^{2}}=0 .
$$

The prescribed boundary conditions Eq.5(a,b) become

$$
\left.\begin{array}{lllll}
\frac{\partial \psi}{\partial X}=0, & T=1, \quad C=1 & \text { at } & Y=0 \\
\frac{\partial \psi}{\partial Y}=1, & T=0, & C=0 & \text { at } & Y \rightarrow \infty,
\end{array}\right\}
$$

where the prime indicates differentiation with respect to $\eta, P e=$ $\frac{U_{\infty} L}{\alpha_{0}}$ represents the Peclet number, $R=\frac{4 \sigma^{*} \bar{T}_{\infty}^{3}}{k^{*} k}$ represents thermal radiation, $B=\frac{\beta_{c}\left(\bar{C}_{w}-\bar{C}_{\infty}\right)}{\beta_{T}\left(\bar{T}_{w}-\bar{T}_{\infty}\right)}$ represents the Buoyancy ratio, $S r=$ $\frac{D_{m} K_{T}}{T_{m} \alpha_{0}}\left(\frac{\bar{T}_{w}-\bar{T}_{\infty}}{\bar{C}_{w}-\bar{C}_{\infty}}\right)$ represents the thermal diffusion, $L e=\frac{\alpha_{0}}{D_{m}}$ indicates the Lewis number, $R a=\frac{L}{\alpha_{0}}\left[\frac{K g \beta_{T}\left(\bar{T}_{w}-\bar{T}_{\infty}\right)}{\nu_{\infty}}\right]^{1 / n}$ represents the generalized Rayleigh number, $\theta_{e}=\frac{\bar{T}_{e}-\bar{T}_{\infty}}{\bar{T}_{w}-\bar{T}_{\infty}}$ indicates the variable viscosity and $\lambda=\left(\frac{R a}{P e}\right)$ indicates the mixed convection parameter.

\section{APPLICATION OF LIE GROUP TRANSFORMATIONS}

We introduce the one-parameter scaling group of transformations which is a simplified form of Lie group transformation

$\Gamma: X^{*}=X e^{\epsilon a_{1}}, Y^{*}=Y e^{\epsilon a_{2}}, \psi^{*}=\psi e^{\epsilon a_{3}}, T^{*}=T e^{\epsilon a_{4}}, C^{*}=C e^{\epsilon a_{5}}$

Here $\varepsilon \neq 0$ is the parameter of the group and the $a_{i}^{\prime} s(I=1,2 \ldots, 5)$ are arbitrary real numbers not all simultaneously zero. Equations (11)(13) along with the boundary conditions (14) do not alter under the group of transformations in Eq.(15) if the $a_{i}$ 's satisfy the following relationship

$$
\left.\begin{array}{l}
n a_{2}-n a_{3}+a_{2}=a_{2}-2 a_{4}=a_{2}-a_{4}-a_{5}=a_{2}-a_{4}=a_{2}-a_{5} \\
a_{1}+a_{2}-a_{3}-a_{4}=2 a_{2}-2 a_{4}=2 a_{2}-a_{4} \\
a_{1}+a_{2}-a_{3}-a_{5}=2 a_{2}-a_{5}=2 a_{2}-a_{4} \\
a_{2}-a_{3}=0 ; \quad a_{4}=0 ; \quad a_{5}=0
\end{array}\right\}
$$

Solving the linear system Eq. (16), we have the following relationship among the exponents:

$$
a_{1}=2 a_{3} ; \quad a_{2}=a_{3} ; \quad a_{4}=0 ; \quad a_{5}=0 .
$$

The transformation $\Gamma$ reduces to

$$
X^{*}=X e^{2 \epsilon a_{3}} ; \quad Y^{*}=Y e^{\epsilon a_{3}} ; \quad \psi^{*}=\psi e^{\epsilon a_{3}} ; \quad T^{*}=T ; \quad C^{*}=C .
$$

Expanding Eq.(18) by Taylor series in powers of $\varepsilon$, and keeping terms up to the first degree (neglecting higher power of $\varepsilon$ ), we obtain

$X^{*}-X=2 \epsilon a_{3} X ; Y^{*}-Y=\epsilon a_{3} Y ; \psi^{*}-\psi=\epsilon a_{3} \psi ; T^{*}=T ; C^{*}=C$.

The characteristic equations are

$$
\frac{d X}{2 X a_{3}}=\frac{d Y}{Y a_{3}}=\frac{d \psi}{\psi a_{3}}=\frac{d T}{0}=\frac{d C}{0} .
$$

Solving the above characteristic equation, we have the following similarity transformations,

$$
\psi=X^{\frac{1}{2}} S(\eta), \quad \eta=Y X^{-\frac{1}{2}}, \quad T=T(\eta), \quad C=C(\eta) .
$$

Substituting Eq. (21) into Eqs. (11) - (13), we obtain the following ordinary differential equations.

$$
\begin{gathered}
n\left(S^{\prime}\right)^{n-1} S^{\prime \prime}=\lambda^{n}\left(T^{\prime}+B C^{\prime}\right)\left(\frac{\theta_{e}-T}{\theta_{e}}\right)-\lambda^{n}(T+B C) \frac{T^{\prime}}{\theta_{e}}, \\
\beta\left(T^{\prime}\right)^{2}+\left(1+T \beta+\frac{4}{3} R\right) T^{\prime \prime}+\frac{1}{2} S T^{\prime}=0, \\
\frac{1}{L e} C^{\prime \prime}+S r T^{\prime \prime}+\frac{1}{2} S C^{\prime}=0,
\end{gathered}
$$

The transformed boundary conditions Eq. (14) become

$$
\begin{array}{ccc}
S(0)=0, & T(0)=1, & C(0)=1 \\
S^{\prime}(\infty)=1, & T(\infty)=0, & C(\infty)=0 .
\end{array}
$$

\section{HEAT AND MASS TRANSFER COEFFICIENTS}

The non-dimensional heat and mass transfer coefficients in terms of Nusselt number $\mathrm{Nu}$ and the Sherwood number $S h$ are respectively given by:

$q_{w}=-k\left(\frac{\partial \bar{T}}{\partial \bar{Y}}\right)_{\bar{Y}=0}-\frac{4 \sigma^{*}}{3 k^{*}}\left(\frac{\partial \bar{T}^{4}}{\partial \bar{Y}}\right)_{\bar{Y}=0} \quad$ and $\quad q_{m}=-D\left(\frac{\partial \bar{C}}{\partial \bar{Y}}\right)_{\bar{Y}=0}$

The Nusselt number $N u=\frac{q_{w} \bar{X}}{k\left(\bar{T}_{w}-\bar{T}_{\infty}\right)}$ and Sherwood number $S h=\frac{q_{m} \bar{X}}{D\left(\bar{C}_{w}-\bar{C}_{\infty}\right)}$ are given by

$$
\frac{N u}{X^{1 / 2}}=-\left(1+\frac{4 R}{3}\right) T^{\prime}(0) \text { and } \quad \frac{S h}{X^{1 / 2}}=-C^{\prime}(0) \text {. }
$$

\section{RESULTS AND DISCUSSIONS}

To solve the system of nonlinear Eqns. (22) - (24) along with the corresponding boundary conditions (25), we apply the Runge-Kutta fourthorder method with a shooting technique, which has been implemented by the many researchers ((Srinivasacharya et al. (2011) and Srinivasacharya et al. (2012))) to solve boundary value problems. In order to validate our code, we compare our obtained results with those of Chaoyang et al. (1990) as a special case by taking $S r=R=B=\beta=0, L e=1.0$, and $\theta_{e} \rightarrow \infty$. Excellent agreement as presented in Tables.1 and Table.2 is obtained.

The results for the non-dimensional velocity, temperature and concentration have been computed and presented graphically in Figs.2-6 to analyze the influence of variable viscosity $\left(\theta_{e}\right)$, thermal conductivity $(\beta)$, radiation $(\mathrm{R})$ Soret parameter $(\mathrm{Sr})$ and mixed convection parameter $(\lambda)$.

Figure 2 displays the dimensionless velocity $S^{\prime}(\eta)$, temperature $T(\eta)$ and concentration $C(\eta)$ distribution in the boundary layer for different 
Table 1 Comparison of Nusselt number for $R=S r=B=\beta=0$, $L e=1.0, \theta_{e} \rightarrow \infty$

\begin{tabular}{|c|cc|}
\hline & $n=0.5$ & \\
\hline$\lambda$ & (Chaoyang et al., 1990) & Present results \\
\hline 0.0 & 0.5641 & 0.564190 \\
0.5 & 0.8209 & 0.821704 \\
1.0 & 0.9303 & 0.929635 \\
4.0 & 1.3010 & 1.300685 \\
\hline
\end{tabular}

Table 2 Comparison of Nusselt number for $R=S r=B=\beta=0$, $L e=1.0, \theta_{e} \rightarrow \infty$

\begin{tabular}{|c|cc|}
\hline & $n=1.5$ & \\
\hline$\lambda$ & (Chaoyang et al., 1990) & Present results \\
\hline 0.0 & 0.5641 & 0.564190 \\
0.5 & 0.6034 & 0.60339 \\
1.0 & 0.6634 & 0.663375 \\
4.0 & 1.0180 & 1.017582 \\
\hline
\end{tabular}

values of the variable viscosity $\left(\theta_{e}\right)$ and power-law index $n$ namely, shear thinning, Newtonian and shear thickening fluids. Figure 2(a) illustrates that velocity $S^{\prime}(\eta)$ enhances near the plate up to a certain value and then reduces far away from the plate with increasing value of variable viscosity for the three types of fluids. The reason for above behavior is that, for a given fluid, when $\gamma$ is fixed, smaller $\left(\theta_{e}\right)$ implies higher temperature difference between the wall and the ambient fluid. From Figs. 2(b) -2(c) we observe that when the variable viscosity is increased there is a reduction in the value of temperature and concentration for the three types of fluids. The reason for this is enhancement in the obstruction of fluid motion to enhance in temperature-dependent viscosity $\left(\theta_{e}\right)$.

The variation of thermal conductivity $(\beta)$ and power-law index $(n)$, namely $(n<1, n=1, n>1)$ for fixed values of the other parameters on dimensionless velocity $S^{\prime}(\eta)$, temperature $T(\eta)$ and concentration $C(\eta)$ profiles in the boundary layer is displayed in Fig. 3. It is observed from Fig. 3(a) that increase in the value of thermal conductivity reduces velocity near the plate and enhances it far away from the plate for the three types of fluids. Figure 3(b) shows that the temperature $\theta(\eta)$ is more pronounced with increasing values of thermal conductivity for the three types of fluids. It is observed that the rise of thermal conductivity results in an increase in the thermal boundary layer thickness. From Fig. 3(c), it is clear that enhancement in the value of thermal conductivity slightly decreases the concentration profile for the three types of fluids. The reason is that molecular motion of the fluid reduces at a slower rate for larger values of $(\beta)$.

The effect of radiation parameter $(R)$ and the power-law index $(n)$ namely, pseudo-plastic fluid, Newtonian, dilatant fluids in the boundary layer is illustrated in Fig.4. Figure 4(a) indicates that an enhancement in the radiation parameter, reduces the velocity $S^{\prime}(\eta)$ slightly near the plate and enhances it far away from the plate for the three types of fluids. In Fig. 4(b) temperature $T(\eta)$ increases monotonically with enhancing values of the radiation parameter $(R)$ for the three types of fluids. This is due to fact that the slope of the temperature distribution near the surface in the presence of $(R)$ is always negative and thus heat is always transferred from the surface. Moreover, Fig. 4(c) shows that for the three different types of fluid concentration $C(\eta)$ reduces slightly with increasing radiation parameter $(R)$.

Figure 5 represents the boundary layer for different values of the Soret parameter $(\mathrm{Sr})$ and power-law fluid index $(n)$, and fixed values of the other parameters. Figure 5(a) shows that the velocity $f^{\prime}(\eta)$ enhances with an enhancement in the Soret parameter $(\mathrm{Sr})$ for the three variations of fluids. Moreover, enhancement in the value of the Soret parameter (Sr) results a slightly raised temperature $T(\eta)$ of the fluid as shown in Fig. 5(b). The influence of Soret parameter $(\mathrm{Sr})$ on the concentration profile $C(\eta)$ is depicted in fig. 5(c). The profile enhancement with enhance in the value of $(\mathrm{Sr})$ for the three variations of fluids. Figure 6 shows the variation of mixed convection parameter $(\lambda)$ and power-law

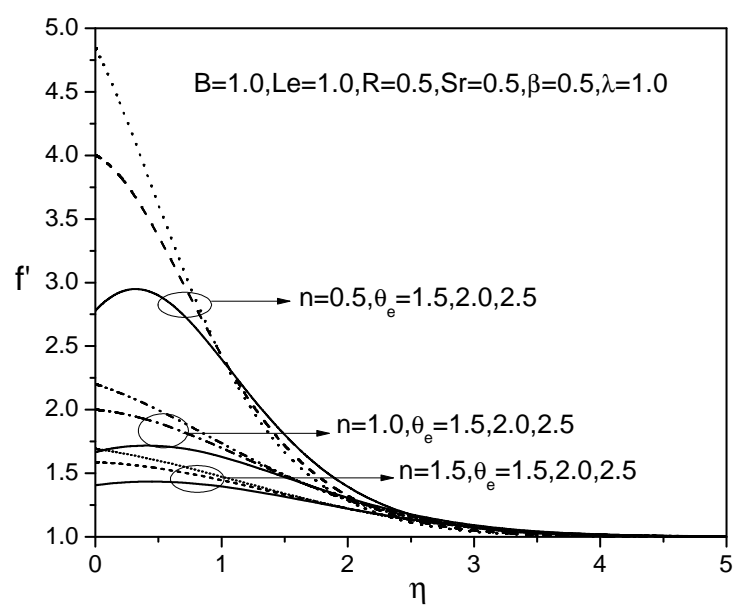

(a)

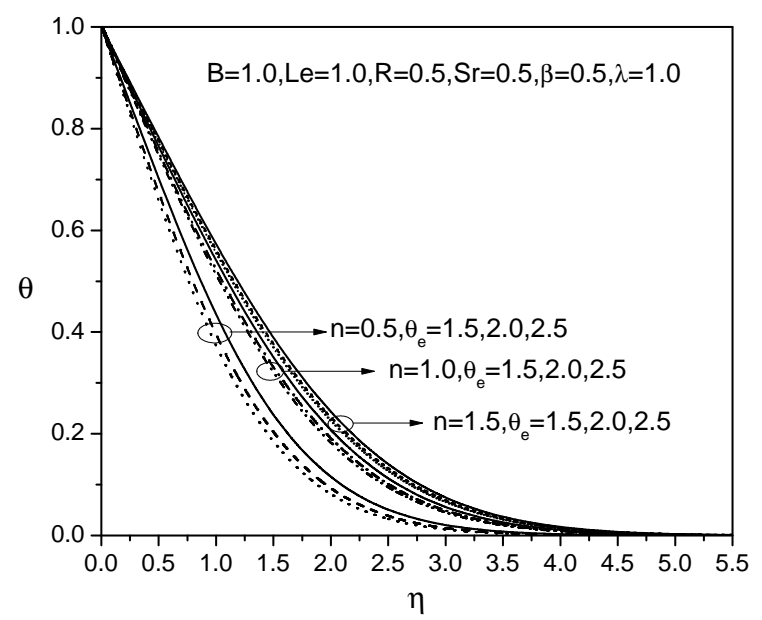

(b)

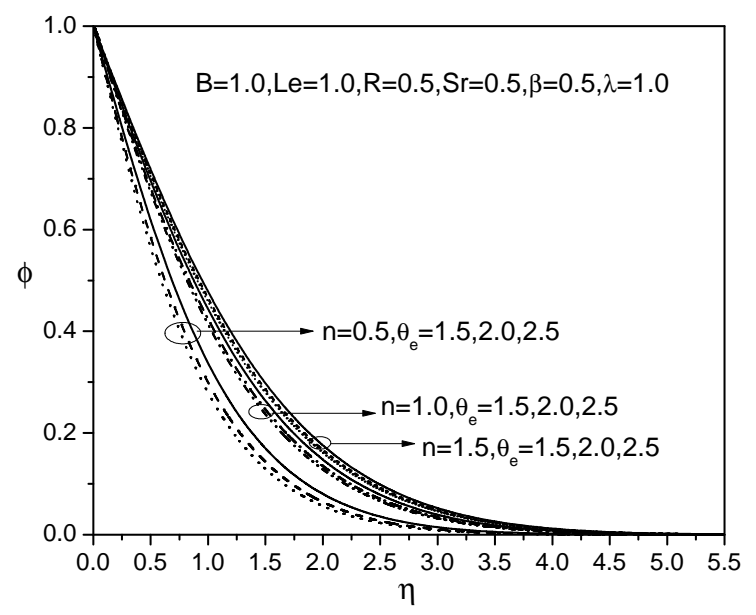

(c)

Fig. 2 Variation of (a) Velocity (b) Temperature and (c) Concentration profiles with variable viscosity $\left(\theta_{e}\right)$. 
index $n$, respectively. From Fig. 6(a) enhancing the value of mixed convection parameter $(\lambda)$ continuously raises the velocity $S^{\prime}(\eta)$ for the three variations of fluids. Figure $6(\mathrm{~b})$ represents that the temperature $T(\eta)$ increases with decrease in the values of the mixed convection parameter $(\lambda)$ for three variations of fluids. Figure 6(c) depicts that the concentra-

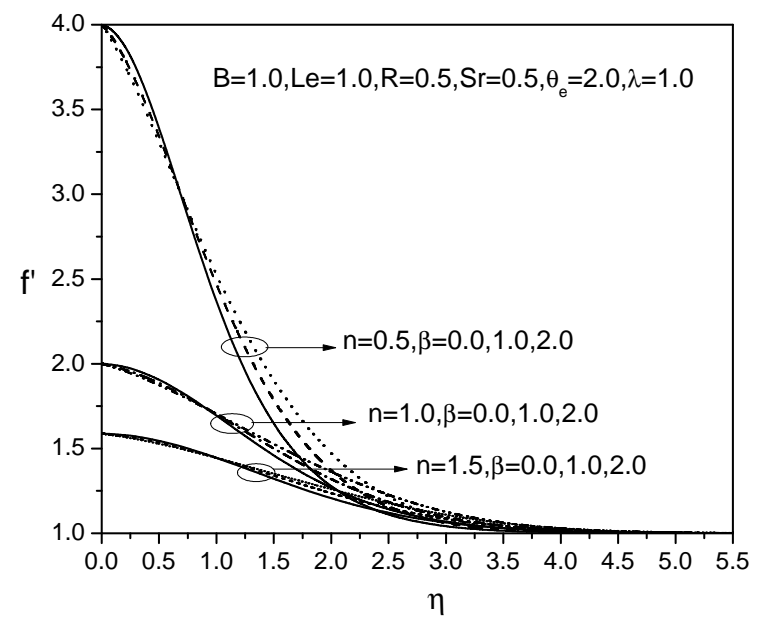

(a)



(b)

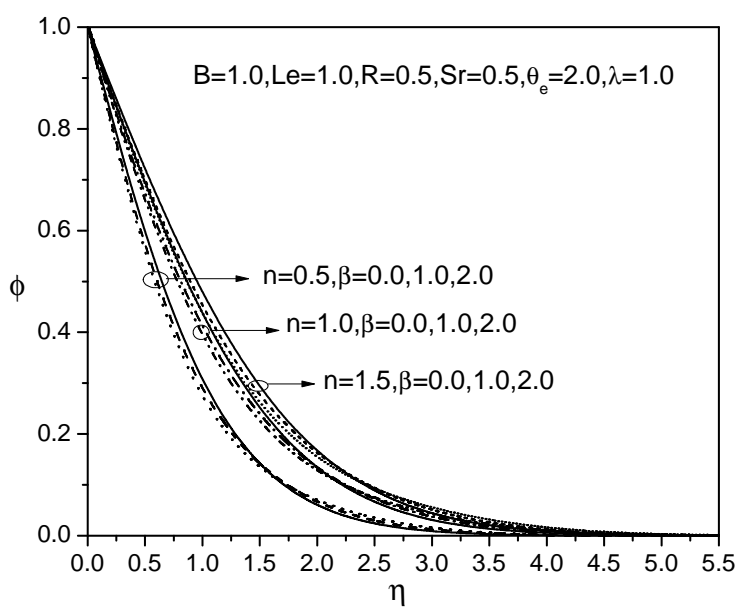

(c)

Fig. 3 Variation of (a) Velocity (b) Temperature and (c) Concentration profiles with thermal conductivity $(\beta)$. tion $C(\eta)$ decreases with an increase in the mixed convection parameter $(\lambda)$ for the three variations of fluids.

Table. 3 shows the influence of heat and mass transfer for various values of the power law index $(n)$, variable viscosity $\left(\theta_{e}\right)$, thermal conductivity $(\beta)$, thermal radiation $(R)$, Soret parameter $(S r)$ and mixed convection parameter $(\lambda)$ for fixed values of the other parameters. En-

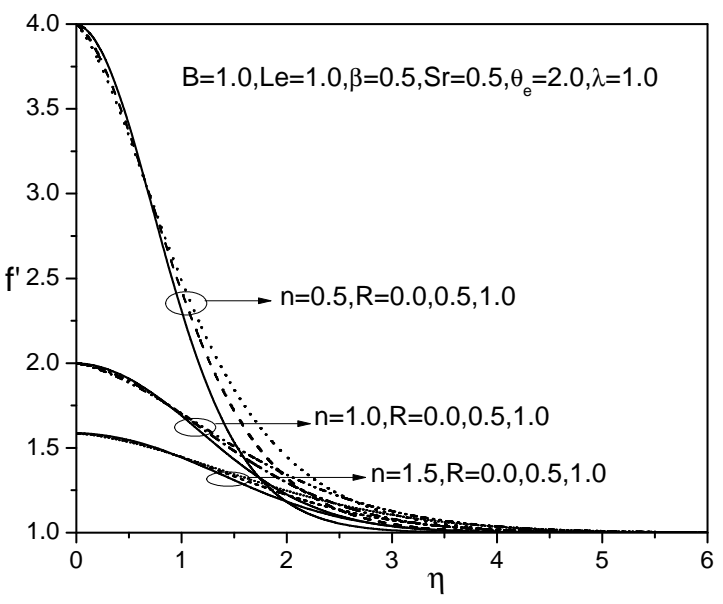

(a)

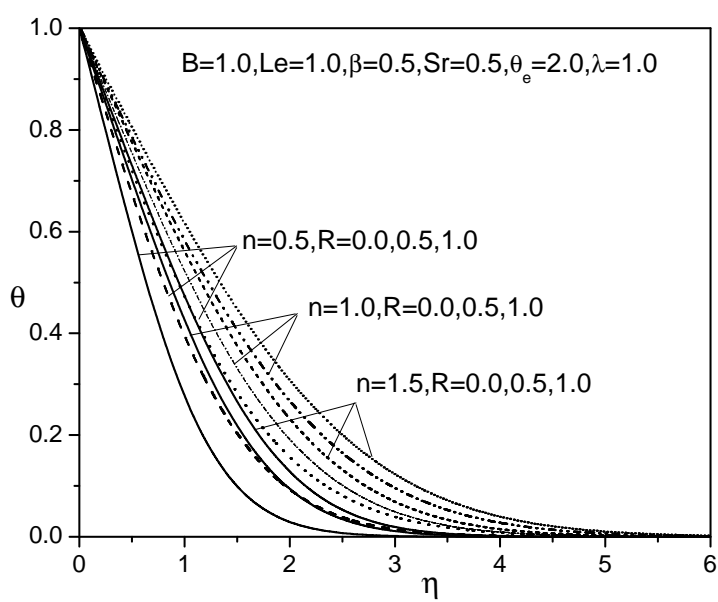

(b)

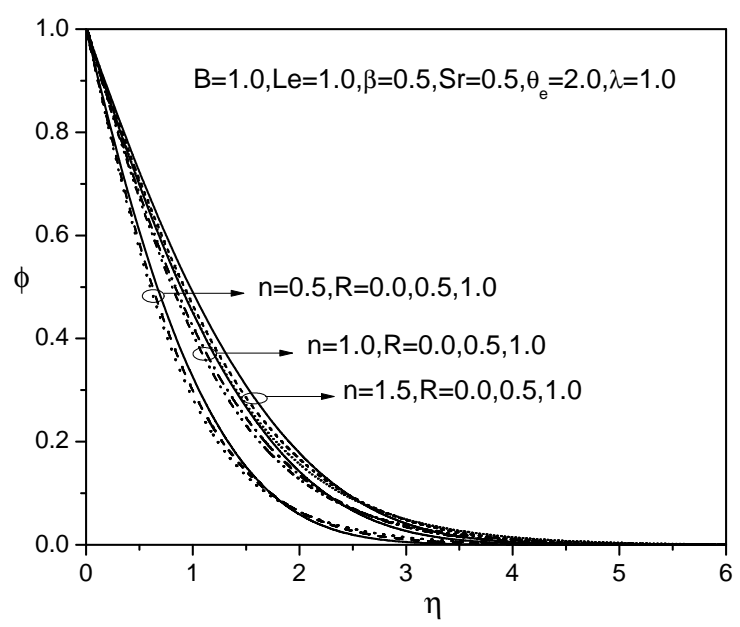

(c)

Fig. 4 Variation of (a) Velocity (b) Temperature and (c) Concentration profiles with radiation parameter $(R)$. 
hancing the value of the $n$ reduces the heat and mass transfer rates. An increase in the values of variable viscosity, radiation and mixed convection parameters raises both the heat and mass transfer rates. It is noticed that an increase in the value of the thermal conductivity decreases the heat transfer, but a reverse trend is observed in the mass transfer rate. Higher

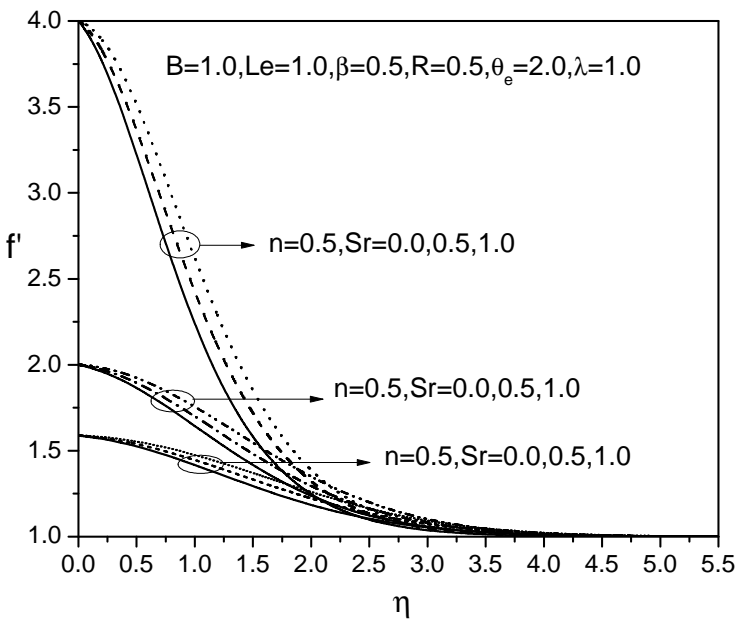

(a)



(b)

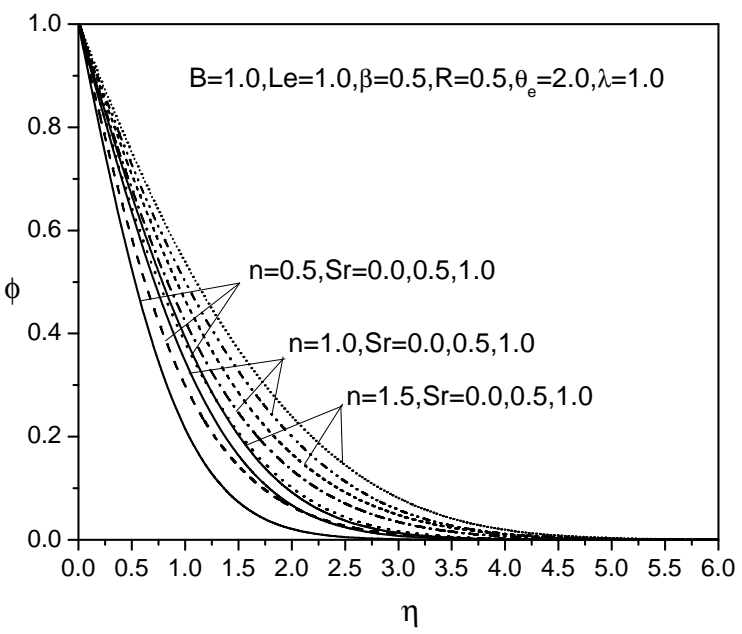

(c)

Fig. 5 Variation of (a) Velocity (b) Temperature and (c) Concentration profiles with Soret parameter $(S r)$. value of the Soret parameter enhance the heat transfer rate and decrease the mass transfer rate.

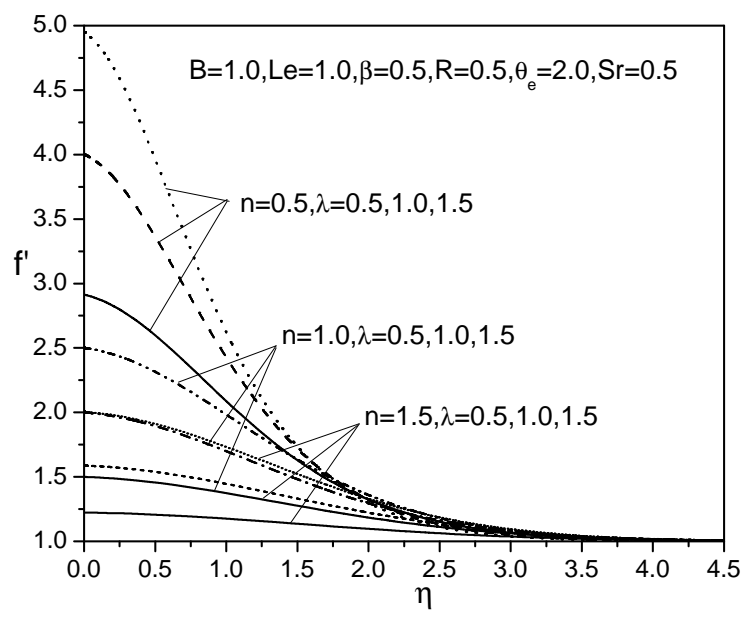

(a)

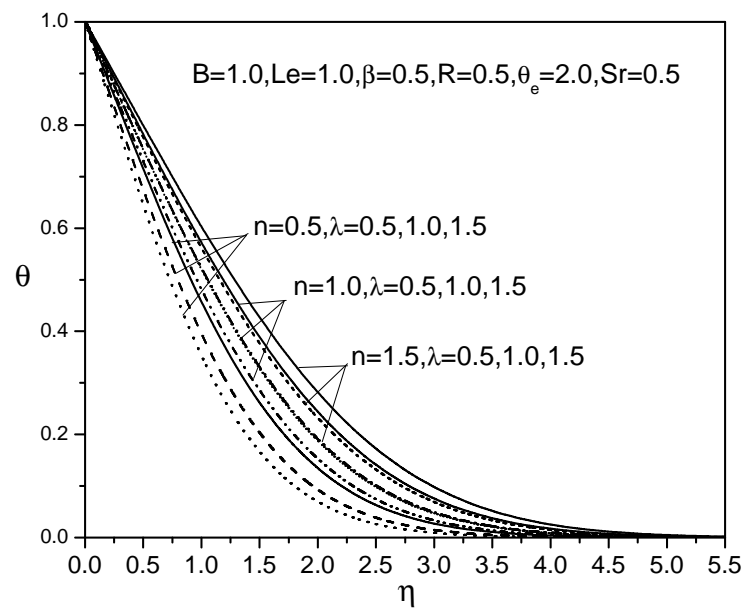

(b)

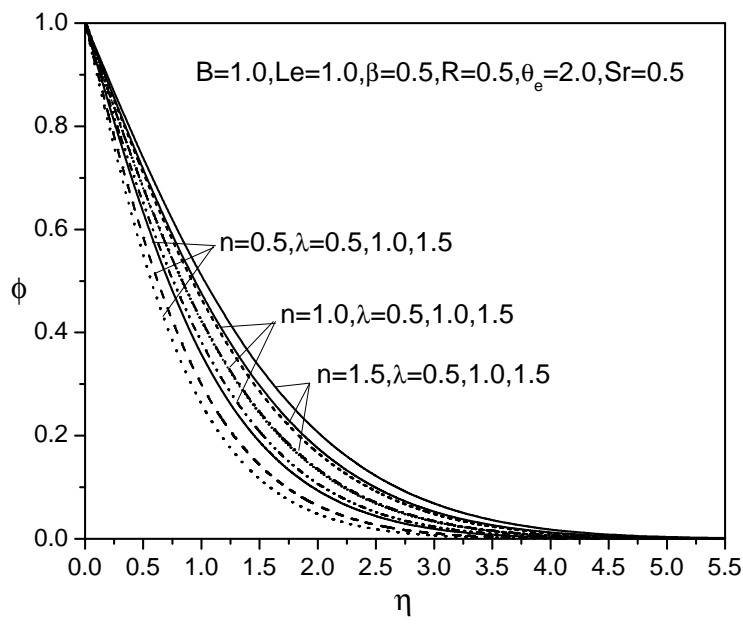

(c)

Fig. 6 Variation of (a) Velocity (b) Temperature and (c) Concentration profiles with mixed convection parameter $(\lambda)$. 
Table 3 Values of heat and mass transfer rates at Le=1.0 and B=1.0 for varying values of power-law index, temperature dependent viscosity, variable thermal conductivity, radiation, Soret and mixed convection parameters.

\begin{tabular}{|cccccc|cc|}
\hline$n$ & $\theta_{e}$ & $\beta$ & $R$ & $S r$ & $\lambda$ & $-T^{\prime}(0)\left(1+\frac{4 R}{3}\right)$ & $-C^{\prime}(0)$ \\
\hline 0.5 & 2.0 & 0.5 & 0.5 & 0.5 & 1.0 & 1.083878 & 0.898871 \\
1.0 & 2.0 & 0.5 & 0.5 & 0.5 & 1.0 & 0.811355 & 0.665762 \\
1.5 & 2.0 & 0.5 & 0.5 & 0.5 & 1.0 & 0.737721 & 0.603112 \\
\hline 0.5 & 1.5 & 0.5 & 0.5 & 0.5 & 1.0 & 0.985773 & 0.810344 \\
0.5 & 2.5 & 0.5 & 0.5 & 0.5 & 1.0 & 1.143469 & 0.952729 \\
0.5 & 3.0 & 0.5 & 0.5 & 0.5 & 1.0 & 1.183445 & 0.988888 \\
\hline 0.5 & 2.0 & 0.0 & 0.5 & 0.5 & 1.0 & 1.305338 & 0.849427 \\
0.5 & 2.0 & 1.0 & 0.5 & 0.5 & 1.0 & 0.939365 & 0.928902 \\
0.5 & 2.0 & 1.5 & 0.5 & 0.5 & 1.0 & 0.836542 & 0.948922 \\
\hline 0.5 & 2.0 & 0.5 & 0.0 & 0.5 & 1.0 & 0.790250 & 0.854522 \\
0.5 & 2.0 & 0.5 & 1.0 & 0.5 & 1.0 & 1.311348 & 0.925503 \\
0.5 & 2.0 & 0.5 & 1.5 & 0.5 & 1.0 & 1.501126 & 0.943220 \\
\hline 0.5 & 2.0 & 0.5 & 0.5 & 0.0 & 1.0 & 1.060429 & 1.028426 \\
0.5 & 2.0 & 0.5 & 0.5 & 1.0 & 1.0 & 1.107032 & 0.768095 \\
0.5 & 2.0 & 0.5 & 0.5 & 1.5 & 1.0 & 1.129908 & 0.636080 \\
\hline 0.5 & 2.0 & 0.5 & 0.5 & 0.5 & 0.5 & 0.945030 & 0.780440 \\
0.5 & 2.0 & 0.5 & 0.5 & 0.5 & 1.5 & 1.190563 & 0.989876 \\
0.5 & 2.0 & 0.5 & 0.5 & 0.5 & 2.0 & 1.280580 & 1.066663 \\
\hline
\end{tabular}

\section{CONCLUSION}

In the present paper, we have analyzed mixed convective flow, heat and mass transfer over a vertical surface in a porous medium saturated with power-law fluid. The numerical results were obtained for different values of the power law index $(n)$, variable viscosity $\left(\theta_{e}\right)$, variable thermal conductivity $(\beta)$, thermal radiation $(\mathrm{R})$ and Soret parameter $(\mathrm{Sr})$. The main conclusions are as follows.

- The variable viscosity parameter $\left(\theta_{e}\right)$ affects the flow field, i.e. enhancement in values of the variable viscosity parameter increases the velocity, heat and mass transfer rates, but decreases the temperature and concentration profiles.

- The effect of variable thermal conductivity $(\beta)$ decreases the velocity, concentration and heat transfer rates, but enhances the temperature and mass transfer rates.

- The effect of the radiation parameter $(R)$ reduces the velocity and concentration profiles, but enhances the temperature, heat and mass transfer rates.

- An increasing value of the Soret parameter $(S r)$ decreases the temperature and mass transfer rates, but monotonically increases the velocity, concentration and heat transfer rates.

- The influence of the mixed convection parameter $(\lambda)$ results in an enhancement in velocity and heat transfer rates, but reduces the temperature, concentration and mass transfer rates.

\section{ACKNOWLEDGEMENTS}

Mr. G. Venkata Suman, the second author thanks the INSPIRE (DST) for financial support in the form of fellowship (No.DST/INSPIRE Fellowship/2013/IF131076), Government of India.

\section{REFERENCES}

Afify, A.A., Uddin, M.J., and Ferdows, M., 2014, "Scaling Group Transformation for MHD Boundary Layer Flow over Permeable Stretching Sheet in Presence of Slip Flow with Newtonian Heating Effects," Applied Mathematies and Mechanics English Edition, 35(11), 1375-1386. http://dx.doi.org/10.1007/s10483-014-1873-7.

Chaoyang, W., Chuanjing, T., and Xiaofen, Z., 1990, "Mixed Convection of non-Newtonian Fluids from a Vertical Plate Embedded in a Porous Medium," Acta Mechanica Sincia, 6(3), 214-220. http://dx.doi.org/10.1007/BF02487642.

El-Aziz, M.A., and Afify, A.A., 2016, "Lie Group Analysis of Hydromagnetic Flow and Heat Transfer of a Power-Law Fluid over Stretching Surface with Temperature-Dependent Viscosity and Thermal Conductivity," International Journal of Modern Physics, 27(11), 1650150. http://dx.doi.org/10.1155/2015/650813.

Elsayed, Elbarbary, M.E., and S.Elgazery, N., 2004, "Chebyshev Finite Difference Method for the Effects of Variable Viscosity and Variable Thermal Conductivity on Heat Transfer from Moving Surfaces with Radiation," International Journal of Thermal Sciences, 43(9), 889-899. http://dx.doi.org/10.1016/j.ijthermalsci.2004.01.008.

Ferdows, M., Uddin, M.J., and Afify, A.A., 2013, "Scaling Group Transformation for MHD Boundary Layer Free Convective Heat and Mass Transfer Flow Past a Convectively Heated nonlinear Radiating Stretching Sheet," International Journal of Heat and Mass Transfer, 56(1), 181187.

http://dx.doi.org/10.1016/j.ijheatmasstransfer.2012.09.020.

Gbadeyan, J.A., Idowu, A.S., Ogunsola, A.W., Agboola, O.O., and Olanrewaju, P.O., 2011, "Heat and Mass Transfer for Soret and Dufours Effect on Mixed Convection Boundary layer Flow over a Stretching Vertical Surface in a Porous Medium Filled with a Viscoelastic Fluid in the Presence of Magnetic Field," Global Journal of Science Frontier Research, 11(8), 97-114.

Grosan, T., and Pop, I., 2006, "A note on the Effect of Radiation on Free Convection over a Vertical Flat Plate Embedded in a non-Newtonian Fluid Saturated Porous Medium," Applied Mechanics and Engineering, 11(3), 715-722.

Hassanien, I.A., Essawy, A.H., and Moursy, N.M., 2003, "Variable Viscosity and Thermal Conductivity Effects on Combined Heat and Mass Transfer in Mixed Convection over a UHF/UMF Wedge in Porous Media: the Entire Regime," Applied Mathematics and Computation, 145(2), 667-682.

http://dx.doi.org/10.1016/S0096-3003(03)00181-4.

Hayat, T., Mustafa, M., and Obaidat, S., 2011, "Simultaneous Effects of MHD and Thermal Radiation on the Mixed Convection Stagnation-Point Flow of a Power-law Fluid," Journal of Applied Mechanics and Technical Physics, 28(7), 074702.

http://dx.doi.org/10.1088/0256-307X/28/7/074702.

Jayanthi, S., and Kumari, M., 2007, "Effect of Variable Viscosity on nonDarcy Free or Mixed Convection Flow on a Vertical Surface in a nonNewtonian Fluid Saturated Porous Medium," Applied mathematics and computation, 186(2), 1643-1659.

http://dx.doi.org/10.1016/j.amc.2006.08.072.

Kairi, R.R., Narayana, P.A.L., and Murthy, P.V.S.N., 2009, "The Effect of Double Dispersion on Natural Convection Heat and Mass Transfer in a Non-Newtonian Fluid Saturated Non-Darcy Porous Medium," Transport in porous media, 76(3), 377-390.

http://dx.doi.org/10.1007/s11242-008-9252-6. 
Kandasamy, R., Muhaimin, I., and Saim, H.B., 2010, "Lie Group Analysis for the Effect of Temperature-Dependent Fluid Viscosity with Thermophoresis and Chemical Reaction on MHD Free Convective Heat and Mass Transfer over a Porous Stretching Surface in the Presence of Heat Source/Sink," Communications in Nonlinear Science and Numerical Simulation, 15(8), 2109-2123.

http://dx.doi.org/10.1016/j.cnsns.2009.09.016.

Lai, E.C., and Kulacki, F.A., 1990, "Effects of Variable Viscosity on Convective Heat Transfer along a Vertical Surface in a Saturated Porous Medium," International Journal of Heat and Mass Transfer, 33(5), 10281031.

http://dx.doi.org/10.1016/0017-9310(90)90084-8.

Mahanti, N.C., and Gaur, P., 2009, "Effects of Varying Viscosity and Thermal Conductivity on Steady Free Convective Flow and Heat Transfer along an Isothermal Vertical Plate in The Presence of Heat Sink," $J$ Applied Fluid Mechanics, 2(1), 23-28.

Mahmoud, M.A.A., and Megahed, A.M., 2013, “Thermal Radiation Effect on Mixed Convection Heat and Mass Transfer of a non-Newtonian Fluid over a Vertical Surface Embedded in a Porous Medium in the Presence of Thermal Diffusion and Diffusion-thermo Effects," Journal of Applied Mechanics and Technical Physics, 54(1), 90-99.

http://dx.doi.org/10.1134/S0021894413010112.

Nakayama, A., 1993, "Free Convection from a Horizontal Line Heat Source in a Power-law Fluid-Saturated Porous Medium,' Int J Heat and Fluid Flow, 14(3), 279-283.

http://dx.doi.org/10.1016/0142-727X(93)90059-V.

Nield, D., and Bejan, A., 2006, Convection in porous media, SpringerVerlag, New York.

Oberlack, M., 1999, "Similarity in non-Rotating and Rotating Turbulent Pipe Flows," Journal of Fluid Mechanics, 379, 1-22.

Pop, I., and Ingham, D., 2001, Convective Heat Transfer: Mathematical and Computational Modelling of Viscous Fluids and Porous media, Elsevier, London.

Rao, P.S., Kumbhakar, B.K., and Rathish, B.V., 2017, "Effect of Thermal Radiation on MHD Flow with Variable Viscosity and Thermal Conductivity Over a Stretching Sheet in Porous Media," Journal of Nanofluids,
6(2), 292-299.

http://dx.doi.org/10.1166/jon.2017.1313.

Seddeek, M.A., and Salama, F.A., 2007, "The Effects of Temperature Dependent Viscosity and Thermal Conductivity on Unsteady MHD Convective Heat Transfer Past a Semi-infinite Vertical Porous Moving Plate with Variable Suction," Computational Materials Science, 40(2), 667682.

http://dx.doi.org/10.1016/j.commatsci.2006.11.012.

Slattery, J.C., 1972, Momentum, Energy and Mass Transfer in Continua, Mc. Graw-Hill chemical engineering series, New York.

Srinivasacharya, D., Mallikarjuna, B., and Bhuvanavijaya, R., 2015, "Soret and Dufour Effects on Mixed Convection along a Vertical Wavy Surface in a Porous Medium with Variable Properties," Ain Shams Engg Journal, 6(2), 553-564.

http://dx.doi.org/10.1016/j.asej.2014.11.007.

Srinivasacharya, D., Pranitha, J., and RamReddy, C., 2011, "Magnetic Effect on Free Convection in a non-Darcy Porous Medium Saturated with Doubly Stratified Power-Law Fluid," Journal of the Brazilian Society of Mechanical Sciences and Engineering, 33(1), 8-14.

http://dx.doi.org/10.1590/S1678-58782011000100002.

Srinivasacharya, D., Pranitha, J., and RamReddy, C., 2012, "Magnetic and Double Dispersion Effects on Free Convection in a Non-Darcy Porous Medium Saturated With Power-Law Fluid," International Journal for Computational Methods in Engineering Science and Mechanics, 13(3), 210-218.

http://dx.doi.org/10.1080/15502287.2012.660231.

Srinivasacharya, D., RamReddy, C., Pranitha, J., and Postelnicu, A., 2014, "Soret and Dufour Effects on Non-Darcy Free Convection in a Power-Law Fluid in the Presence of a Magnetic Field and Stratification," Heat Transfer-Asian Research, 43(7), 592-606. http://dx.doi.org/10.1002/htj.21098.

Uddin, M., Kabir, M., and Alginahi, Y., 2015, "Lie Group Analysis and Numerical Solution of Magnetohydrodynamic Free Convective Slip Flow of Micropolar Fluid over a Moving Plate with Heat Transfer," Computers and Mathematics with Applications, 70(5), 846-856. http://dx.doi.org/10.1016/j.camwa.2015.06.002.

Vafai, K., 2015, Handbook of Porous Media, Crc Press, London. 